\title{
Fabrication of polymeric substrates with micro- and nanoscale topography bioimprinted at progressive cell morphologies
}

\author{
Lynn M. Murray, Volker Nock, ${ }^{\text {a) }}$ and Maan M. Alkaisi \\ Department for Electrical and Computer Engineering, The MacDiarmid Institute for Advanced Materials \\ and Nanotechnology, University of Canterbury, Private Bag 4800, Christchurch 8140, New Zealand
}

Joanne J. M. Lee and Tim B. F. Woodfield

Department of Orthopedic Surgery and Musculoskeletal Medicine, University of Otago, P.O. Box 4345 , Christchurch 8140, New Zealand

(Received 29 June 2012; accepted 24 September 2012; published 11 October 2012)

\begin{abstract}
This work introduces a novel process for the fabrication of polymer cell culture substrates containing physical topography based on timepoint specific cell phenotype replicas. Bioimprinting of human nasal chondrocyte at different cell adhesion time points was used to demonstrate the nanoscale replication process. Atomic force microscopy confirmed morphology progression at $1,6,12$, and $24 \mathrm{~h}$ timepoints corresponding to dedifferentiation of the chondrocytes to fibroblast-like phenotype. Topographical analysis of the imprinted substrates yielded an inverse relationship between surface coverage, increasing from $11 \%$ to $87 \%$, and maximum average pattern depth, decreasing from $1.2 \mu \mathrm{m}$ to $430 \mathrm{~nm}$. Methacrylate bioimprint features were successively replicated into a transitional polydmethylsiloxane mold used as an intermediate for further replication into polystyrene by a highthroughput embossing method. Substrates fabricated with this process have applications in stem cell engineering, regenerative medicine, and implantable devices. (C) 2012 American Vacuum Society. [http://dx.doi.org/10.1116/1.4758759]
\end{abstract}

\section{INTRODUCTION}

Mechanotransduction in relation to substrate stiffness ${ }^{1,2}$ and topography ${ }^{3,4}$ has been found to play a significant role in cell development and tissue formation. Patterning of surfaces, and nanopatterning, ${ }^{5,6}$ in particular, has been shown to provide a useful tool to influence the adhesion, spreading, morphology and, in case of stem cells, the differentiation outcome of biological cells. One particular application in extensive need of such passively bioactive surfaces is cartilage repair. Within the body, cartilage cells (chondrocytes) are embedded within a spongy, elastic extracellular matrix and maintain a spherical phenotype. For regenerative research applications, chondrocytes are isolated from primary biopsy and thus available only in very limited numbers. ${ }^{7}$

In vitro expansion cell culture is required to increase population numbers. However, chondrocytes expanded on two dimensional polystyrene in traditional tissue culture flasks adhere and spread, expressing a more "fibroblastic" phenotype than presented by chondrocytes in vivo. ${ }^{8}$ This altered morphology and related genetic matrix expression mean that the resulting expanded chondrocytes are less than ideal for potential clinical applications. It is thus of great importance to develop culture substrates which better mimic the natural microenvironment and prevent chondrocytes from redifferentiating into the fibroblastic stage.

Previous work has shown the importance of substrate surface properties on chondrocyte growth and morphology., In particular, gas plasma modification of co-polymer substrates was found to negatively impact chondrocyte redifferentiation. Thus, in this paper, we propose a purely

${ }^{a)}$ Electronic mail: volker.nock@canterbury.ac.nz topographical approach to control cell morphology. This approach relies on nanopatterning of the substrate surface alone and is applicable to standard cell-culture materials such as polystyrene (PS) to provide higher throughput and more widespread availability. By negating the need for chemical surface modification and as a result of their passive nature, physical surface patterns are ideally suited for mass integration into existing polymer cell-culture dishes. As opposed to standard nanofabrication technologies, which are typically limited to regular geometric patterns, we propose the use of bioimprint to form the surface pattern.

Bioimprint substrates contain highly detailed replications of cell features accurate to less than $20 \mathrm{~nm},{ }^{10,11}$ thus physically mimicking the complex natural cellular microenvironment and instantaneous morphology of the cell. In this work, we show for the first time a process for the fabrication of methacrylate-based substrates imprinted with cell morphologies at progressing timepoints during subculture adhesion and early growth. Figure 1 shows a schematic overview of the process steps. The bioimprint samples formed using this process capture chondrocyte morphology at $1,6,12$, and $24 \mathrm{~h}$ and demonstrate the cells' tendency to dedifferentiated and spread. To eliminate any surface chemistry effects on the cells from the methacrylate co-polymer used for its high replication resolution in the primary imprint, we further demonstrate the successful transfer of the combined microand nanoscale features into PS substrates by hot-embossing. Substrates with biomimetic patterns as created by this process have the potential to influence cell development and differentiation outcomes on a purely physical basis and biological experiments to confirm this hypothesis are currently underway. 
a) Fix cells and remove stencil

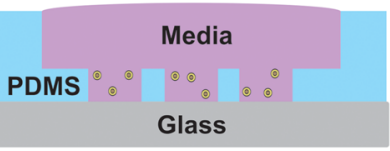

b) Dispense methacrylate

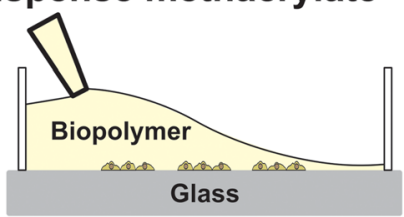

c) UV curing for $20 \mathrm{~s}$ at $100 \mathrm{~W}$

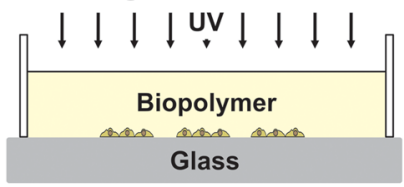

d) Peel-off and image

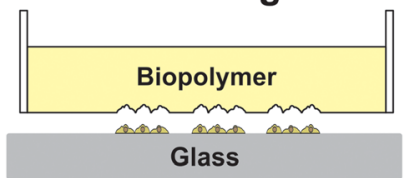

e) Replicate into PDMS

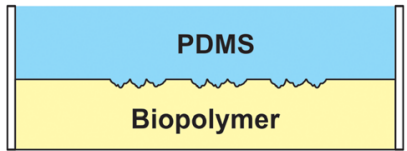

f) Hot emboss into PS sheet $\downarrow \downarrow$ Pressure, $120^{\circ} \mathrm{C} \downarrow \downarrow$

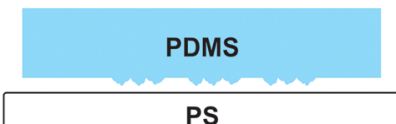

g) Culture secondary cells

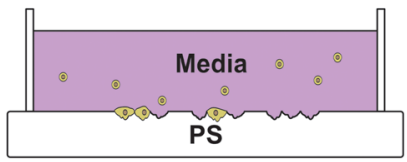

FIG. 1. (Color online) Schematic of the substrate patterning process. (a) Cells are cultured inside PDMS stencils. (b) At various timepoints, cells are fixed, stencils removed, and liquid biopolymer is dispensed onto the cells. (c) The polymer is UV-cured and (d) peeled off the cells. (e) Alternatively, a PDMS replica is formed of the polymer imprint and (f) transferred into a PS substrate via hot-embossing. (g) Patterned PS substrates are then used as culture substrates.

\section{EXPERIMENT}

\section{A. Culture device fabrication}

Polydimethylsiloxane (PDMS) cell culture stencils were fabricated using exclusion molding. ${ }^{5}$ In brief, a laser mask writer ( $\mu$ PG101, Heidelberg Microsystems) was used to transfer the stencil design onto chrome-glass masks. These were used to expose SU-8 2100 (Microchem, USA) spincoated to $200 \mu \mathrm{m}$ thickness onto a $100 \mathrm{~mm}$ silicon wafer. After development, the SU-8/Silicon mold was treated with a release agent (Tetramethylchlorosilane, Sigma). PDMS prepolymer (Sylgard 184, Dow Corning) was mixed at a 10:1 w/w ratio, degassed, and poured onto the mold. Excess polymer was excluded from the mold using a multilayer assembly and a $3 \mathrm{~kg}$ weight. ${ }^{12}$ The stack was cured for $2 \mathrm{~h}$ at $80^{\circ} \mathrm{C}$ on a hotplate. After curing, the PDMS stencils were peeled-off the mold. Macroscale cell culture chambers were cut from a $2 \mathrm{~mm}$ thick flat slab of cured PDMS using a $14 \mathrm{~mm}$ hole punch. Both stencils and wells were sterilized using UV light prior to use.

\section{B. Cell culture}

Human nasal chondrocytes were obtained from primary biopsy at Christchurch Hospital. Chondrocytes were isolated from primary tissue before either expansion cell culture or liquid nitrogen storage for future use. Freshly isolated cells were designated passage zero. Expansion cell culture was maintained in $75 \mathrm{~cm}^{2}$ tissue culture flasks in supplemented Dubelcco's minimum essential media with Glutamax (DMEM) (No. 10569, Life Technologies Corporation). Base DMEM was supplemented with $1 \%$ penicillin streptomycin, $1 \%$ nonessential amino acids (NEAA), HEPES (0.01 M final concentration), and L-proline (.4 $\mathrm{mM}$ final concentration). Additionally, $10 \%$ fetal calf serum (FCS) and 1\% ascorbic acid were added immediately before the first use. Flasks were incubated in $5 \% \mathrm{CO}_{2}$ at $37^{\circ} \mathrm{C}$ for 7 days or until approximately $80 \%$ confluence. Due to the importance of maintaining a low passage number, higher confluence was preferential to subculture. Notably, twin flasks were maintained for each passage to ensure that chondrocytes from the same passage used for both the bioimprint and the secondary culture.

Where applicable, prefabricated PDMS stencils were cut from thin, exclusion-molded PDMS sheet and placed concentrically within PDMS culture chambers. Chondrocyte cells were seeded onto glass within PDMS-defined chambers at $1.0 \times 10^{4}$ cells $/ \mathrm{cm}^{2}$. Slides were removed at $1,6,12$, and $24 \mathrm{~h}$ timepoints. The cells were then fixed with $4 \%$ paraformaldehyde for $45 \mathrm{~min}$, washed twice with PBS, and washed again with deionized water to remove any dissolute salts from the PBS wash. Fixed cells were stored in $4{ }^{\circ} \mathrm{C}$ and allowed to dry for at least $2 \mathrm{~h}$ before bioimprinting to ensure highest replication resolution.

\section{Bioimprint}

The bioimprinting liquid prepolymer consisted of ethylene glycol dimethacrylate, methacrylic acid (both Sigma Aldrich, St. Louis, MO), and IRGAcure 2022 (CIBA Specialty Chemicals, Basel, Switzerland) combined in a 6:3:1 ratio. To prevent undesired crosslinking, the liquid polymer was mixed in a yellow room immediately prior to curing and protected from normal light and UV until curing. Where applicable, the PDMS stencils were removed before $200 \mu \mathrm{l}$ of polymer was pipetted into each PDMS-defined chamber and allowed to settle for a few seconds before curing. Slides with liquid polymer were then exposed to UV for $2 \mathrm{~min}$ (100 W Hg arc lamp, $40 \%$ iris setting, 250-450 nm filter, EXFO Photonic Solutions, Inc., Singapore). 
After curing, solidified polymer replicas were peeled off the cells and left for $24 \mathrm{~h}$ at room temperature in ambient light to further crosslink. Bioimprints were cleaned before characterization and further replication to remove or deactivate possible remaining biological debris and monomers. Bioimprints were washed with deionized water, sonicated in lysis buffer consisting of $10 \%$ SDS in $0.01 \mathrm{~N} \mathrm{HCl}$, and soaked for $45 \mathrm{~min}$ in trypsin. Bioimprints were then soaked for at least $24 \mathrm{~h}$ in deionized water to remove any unreacted acidic monomers. After being removed and dried, bioimprint samples were placed imprint side up within 2-chamber LabTek Chamber Slide system chambers (No. 177429, Nunc) to restrict the outside boundary for the PDMS molding.

Analysis of the bioimprints across the four timepoints was carried out by optical and atomic force microscopy (AFM, Digital Instruments 3100 Nanoscope III, Vecco). Surface coverage was determined by defining the cell boundaries in IMAGEJ (ImageJA, v.1.46 m: http://pacific.mpi-cbg.de/ wiki/index.php/Fiji) and measuring the selected area as a percentage of the overall area. Image area was defined by calibration images taken of known length scales and IMAGEJ scales were defined accordingly. Maximum cell heights were determined for each cell in the AFM images of Fig. 2(b). The maximum cell heights for each time point were averaged and plotted in comparison to the surface coverage.

\section{PS hot embossing}

Methacrylate bioimprints were removed from the postfabrication water bath and allowed to dry thoroughly before a replica mold was created in PDMS. Prepolymer PDMS was poured over the substrate surface containing the bioimprint features. The entire construct was desiccated until there were no bubbles remaining in the liquid PDMS. The PDMS was cured by baking at $80^{\circ} \mathrm{C}$ for $2 \mathrm{~h}$ on a hotplate. Careful separation was required to remove the PDMS from the methacrylate template.

Sheet polystyrene was cut to the approximate dimensions of a microscope slide and sandwiched against the bioimprinted PDMS mold by two glass microscope slides and compressed using medium-sized bulldog clamps. ${ }^{13}$ The glass/styrene/PDMS/glass assembly was placed in a $185^{\circ} \mathrm{C}$ oven for $10 \mathrm{~min}$ and then allowed to cool for at least $1 \mathrm{~h}$ before disassembly.

\section{RESULTS AND DISCUSSION}

Physical morphology is an important indicator of cell health and development. Permanent replication of time dependent cellular features for high resolution offline analysis can help to understand fundamental concepts involved in determining the final cell phenotype. In addition to aiding analysis, the formation of rigid copies of cell morphology provides one with a template for replication into other engineering materials for applications ranging from two dimensional polymer substrates used for expansion cell culture to more complex three dimensional implant surfaces.

\section{A. Bioimprint capture of time-dependent cell morphology}

The capability of the process to capture time-dependent cell morphological changes was initially tested by imprinting human nasal chondrocytes at four representative cell-culture timepoints. These timepoints were chosen to reflect different stages of dedifferentiation observed during the expansion of chondrocyte cells in vitro. Optical imaging of the bioimprinted samples, shown in Fig. 2(a), demonstrates the successful replication of drastically different cell adhesive
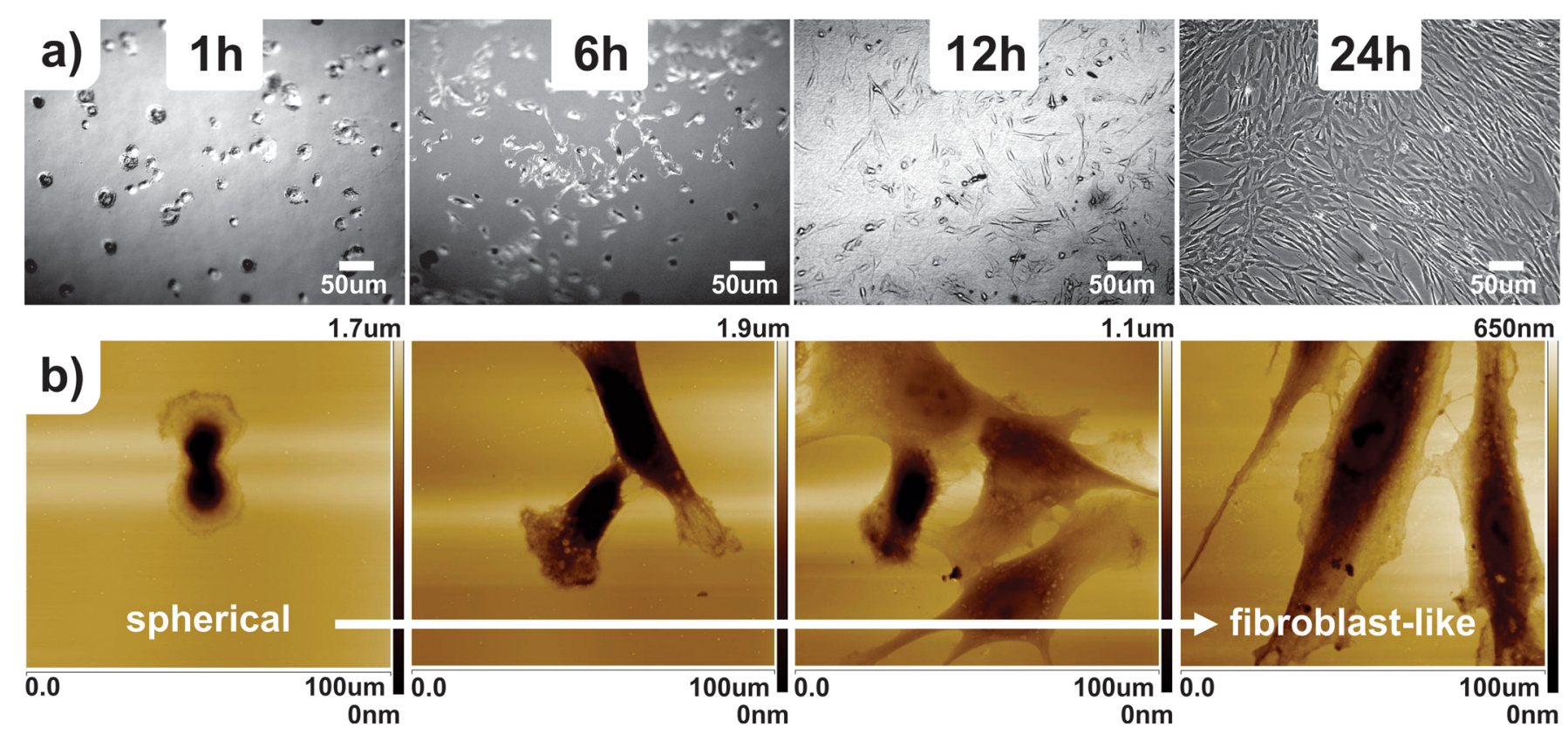

$650 \mathrm{~nm}$

FIG. 2. (Color online) Micrographs showing bioimprints replicated at four timepoints. (a) Brightfield microscopy of methacrylate substrates imprinted off chondrocyte cell cultures fixed at 1, 6, 12, and 24 h timepoints. (b) AFM close-ups of the respective surfaces showing combined micro- and nanoscale feature detail. Cells can be seen to undergo a dedifferentiation process from spheroidal to fibroblast-like morphology, a process which hinders large-scale in vitro expansion of chondrocytes. 
morphologies into rigid methacrylate polymer at $1,6,12$, and $24 \mathrm{~h}$.

Bioimprints taken at only $1 \mathrm{~h}$ after initial cell contact with the surface regularly show a spherical morphology with large lamellipod extrusions. High-resolution atomic force microscopy of the imprints revealed that cells replicated at $1 \mathrm{~h}$ consisted primarily of a central, spherical bulk with a large aspect ratio [see Fig. 2(b)]. While the latter indicates the excellent replication capabilities of the process, the large pattern depth was found to make accurate imaging of the central features with the AFM impossible. However, successfully imaged thick, lamellipod extensions in the cell membrane surrounding the spherical center indicated the spreading of the cells and motility across the substrate surface. The dispersed, spherical potholes of the $1 \mathrm{~h}$ samples reflect the cells as they had only recently adhered.

After only $6 \mathrm{~h}$ of adhesion time in incubation, the bioimprinted cells showed the extension of the membrane protrusions and the cell spreading, but notably cells maintained a high, dense central region which had not yet flattened out across the surface. As expected of an intermediate timepoint, cells at $6 \mathrm{~h}$ expressed a developing morphology and existed in a range of spreading states, which resulted in reduced imprint depth visible in Fig. 2(b).

Bioimprints taken at 12 and $24 \mathrm{~h}$ show similar, highly spread cell morphologies but vary greatly in the number of cells depicted in the substrate. Comparing the micrographs from these two time points [Fig. 2(a)], one can observe significantly higher surface coverage on the $24 \mathrm{~h}$ bioimprint sample. AFM images for both latter timepoints [Fig. 2(b)] show clearly visible nuclear features and highly spread membrane surface areas. It is evident from these sequential AFM images that the cells incur an increasingly spread, fibroblastic morphology, a dynamic transition which the bioimprint process was able to capture into rigid polymer in high detail.

Elastomeric PDMS stencils used to guide the macroscopic cell arrangement during culture showed little effect on the organization of the initial cells at the shorter timepoints (1 and $6 \mathrm{~h})$. The stencil-defined areas were not completely filled with cells at these timepoints, as suggested by the lower surface coverage values. At 12 and $24 \mathrm{~h}$, the stencil borders were at least partially defined by the confluence of cell growth within the borders. The stenciled area was easily identifiable on the resulting bioimprints and subsequent polystyrene replica. The bioimprint-patterned substrate can subsequently be used as a secondary cell culture substrate containing combined lithographic patterning and nanoscale cell features. To better understand the nature of the cell-substrate interaction and its effect on cell development, further quantitative surface characterization was conducted.

Topographical analysis of the timepoint micrographs showed highly correlated trends for both increasing surface coverage and decreasing maximum feature depth over $24 \mathrm{~h}$. Percentage surface coverage and maximum cell height were plotted across all four timepoints, as shown in Fig. 3. Both surface area coverage and maximum cell height showed highly correlated behavior across $24 \mathrm{~h}$. The small, circular cell morphologies on bioimprints taken at the $1 \mathrm{~h}$ timepoint

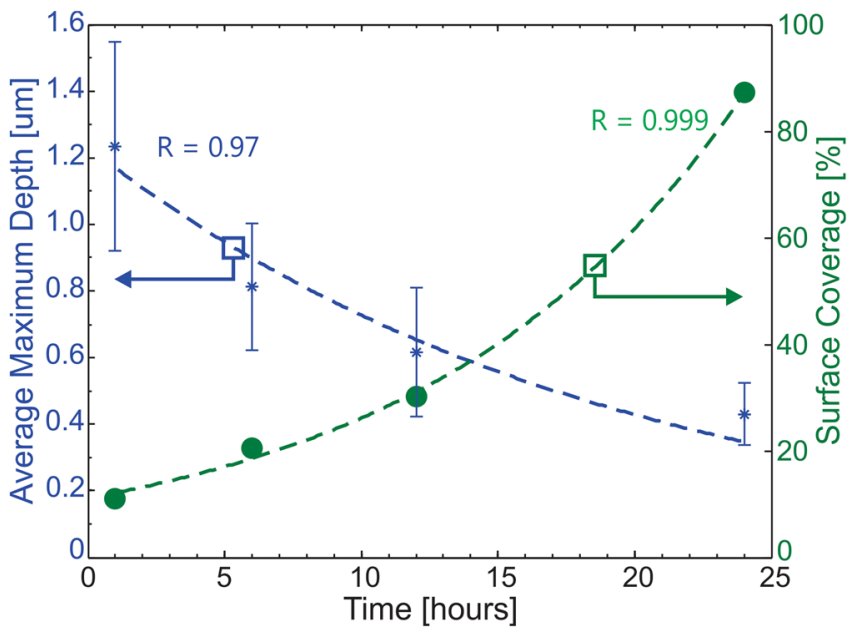

FIG. 3. (Color online) Plot of substrate surface analysis results at different imprint timepoints. Surface coverage increases exponentially from samples imprinted at $1 \mathrm{~h}$ to those imprinted at $24 \mathrm{~h}$. Simultaneously, average maximum pattern depth decreases as chondrocyte morphology changes from spheroidal to fibroblast-like during spreading.

cover only $11 \%$ of the total surface. Surface coverage increases exponentially over the allotted time to an almost fully confluent $87.2 \%$ coverage at $24 \mathrm{~h}$. Similarly, as the cells spread and cover more of the available substrate, the initial spherical cell flattens out. As expected, the cell feature depth was greatest for the freshly adhered cells, approximately $1.2 \mu \mathrm{m}$, and dropped to almost a third of that, $430 \mathrm{~nm}$, by $24 \mathrm{~h}$ (Fig. 3).

The difference in surface coverage between the 1 and $24 \mathrm{~h}$ bioimprint substrates may influence the extent of cell adhesion to the methacrylate imprints. Bioimprints taken at $1 \mathrm{~h}$ still contain mostly flat methacrylate surface with dispersed cell indentations, whereas bioimprints taken later contain a higher percentage of surface coverage with a spread, developed morphology. Surface roughness, which increases with the coverage percentage of the bioimprint sample, may be an important substrate property for adhesion analysis. The timepoint progression shows variation in bioimprint feature density, which is related to cell seeding density for the initial culture and increases with cell spreading. Experiments comparing the effect of bioimprint feature density with regular, lithographically defined features on cell adhesion and morphology are currently underway.

An additional consideration before application as a cell culture substrate is exposed surface chemistry of the methacrylate substrates. Methacrylate-based polymers are well documented as being mildly cytotoxic ${ }^{14}$ and low-adhesive cell substrates. ${ }^{15} \mathrm{We}$ use methacrylate because it has previously demonstrated extremely high resolution replication capabilities. ${ }^{16}$ The specific methacrylate combination used for this work however is not ideal for direct culture because of the exposed hydroxyl groups contributed by the methacrylic acid monomers and the increased cytotoxicity of the short-chain dimethacrylate chemistry supplied by ethylene glycol dimethacrylate incorporation. ${ }^{14}$ To eliminate the effects of methacrylate surface chemistry on cell adhesion and development a further replication of the primary imprint into a "cell-friendly" 
material was performed, results of which are shown in the following.

\section{B. Secondary replication into PS by hot-embossing}

Polystyrene is pervasive throughout biological fields and the traditional substrate for expansion culture. The availability of bioimprinted polystyrene substrates would represent a significant advancement in the definition of this useful technology and its use as an effective cell scaffold. Cell imprints cannot be easily nanopatterned or directly replicated into polystyrene because of solvent chemistry. Therefore, an indirect method for polystyrene embossing was used: transferring the high resolution features of the primary methacrylate imprint into a reusable intermediate PDMS mold. During formation of this PDMS mold, we observed that remaining methacrylate monomers leaching from the bulk polymer can cause incomplete PDMS curing. It was found that a premolding water soak, to remove any water soluble methacrylic acid monomers, encouraged complete PDMS curing and high resolution replication. The PDMS mold combined micro- and nanoscale cell morphologies which, in combination with hot embossing, were successfully transferred into polystyrene. The stenciled bioimprints taken at $24 \mathrm{~h}$, shown in both PDMS and polystyrene in Fig. 4, display incomplete confluence but obviously demarcate the bioimprinted regions with cell-defined boundaries.

Distortion artifacts were seen when the polystyrene was not heated for the full $10 \mathrm{~min}$. This was likely a combined effect of local temperature and pressure gradients. The
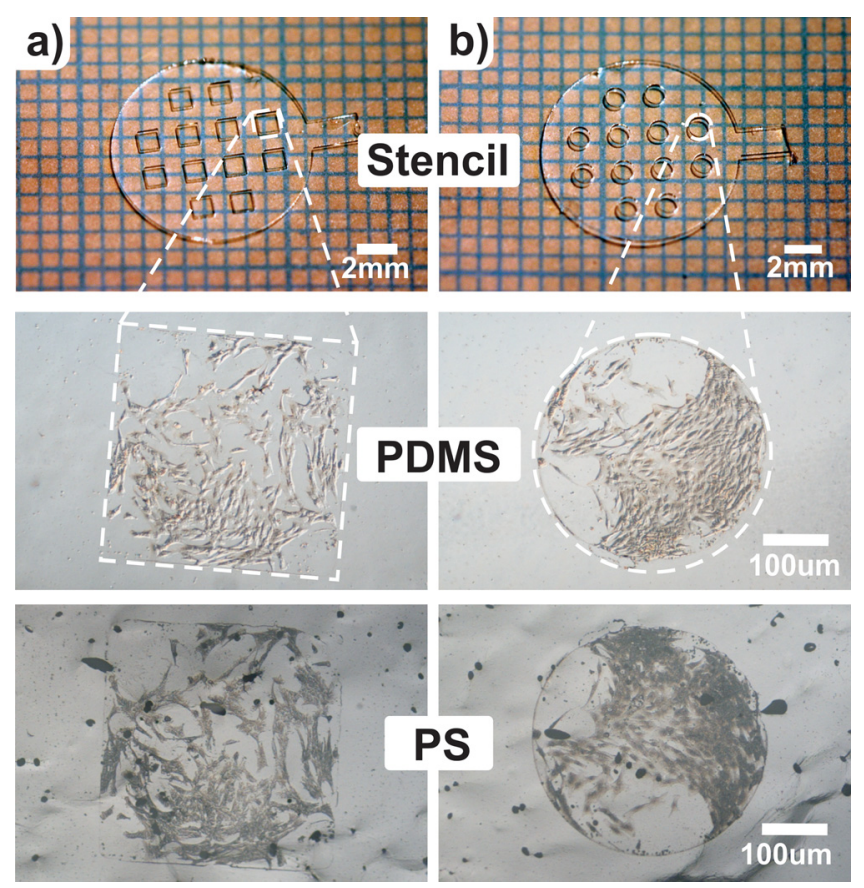

FIG. 4. (Color online) Micrographs of secondary replicas formed by pattern transfer using intermediate PDMS stamps. Square (a) and round (b) PDMS stencils were used to produce the initial negative biopolymer replica. A secondary replication of this bioimprint into PDMS was used as a positive stamp to hot-emboss the cell topography into cell-culture grade PS. The polarity of the latter is equivalent to the initial imprint off the cells, i.e., negative. localized pressure of the bulldog clips prevented uniform melting at shorter heating durations. The overall simplicity of the method and the minimization of gradient effects at high heat, however, showed the advantages to this fabrication protocol. Local variation in polymer mixing and, therefore, surface chemistry of the methacrylate imprint can be circumvented by transferring the detailed cell features into polystyrene substrates. Experiments are underway to compare alternative solvent casting based replication methods from the PDMS bioimprint mold. ${ }^{17}$

Once the PDMS mold was fabricated from the original bioimprint surfaces, the substrate throughput was significantly increased. Sacrificial cell cultures were no longer necessary for every bioimprint fabrication. This is important for disposable, low cost cell culture-ware applications in which consistent surface chemistry and high-throughput fabrication are critical. Combining the PDMS stencils prior to bioimprinting with the polystyrene replication method produced a substrate with well-defined bioimprinted regions (Fig. 4). The resulting substrates contain biologically relevant cell features in defined regions in a substrate with known, homogeneous surface chemistry with the added benefit of increased throughput. We are currently investigating the use of these substrates for cell-substrate adhesion investigations in comparison with lithographically patterned and commercial, unpatterned PS cell culture dishes.

\section{SUMMARY AND CONCLUSIONS}

We have shown the successful replication of human nasal chondrocytes into methacrylate polymer. Timepoint analysis of these bioimprints precisely documented the morphology progression as these cells interact with two dimensional growth substrates. Atomic force microscopy of the bioimprints verified the progressive cell spreading and further demonstrated the high resolution of this nanoimprinting technology. Surface coverage and maximum cell depth data obtained showed an inverse relationship between spreading and depth. Imprinted, biomimetic patterns with highly ordered geometric regions were transferred successfully into biologically relevant polystyrene substrates, thus enabling widespread uptake of this technology into conventional cell culture protocols.

\section{ACKNOWLEDGMENTS}

The authors would like to thank Ben Schon, Helen Devereux, and Gary Turner for technical assistance. Financial support for V. Nock and L. M. Murray was provided by Marsden fund Contract No. UOC-1006. J. J. M. Lee was supported by the Royal Society of New Zealand Canterbury Branch through a summer scholarship.

\footnotetext{
${ }^{1}$ A. J. Engler, S. Sen, H. L. Sweeney, and D. E. Discher, Cell 126, 677 (2006).

${ }^{2}$ D. E. Discher, P. Janmey, and W. Yu-li, Science 310, 1139 (2005).

${ }^{3}$ M. Nikkhah, F. Edalat, S. Manoucheri, and A. Khademhosseini, Biomaterials 33, 5230 (2012).

${ }^{4}$ K. Kulangara, Y. Yang, J. Yang, and K. W. Leong, Biomaterials 33, 4998 (2012).
} 
${ }^{5}$ R. Kriparamanan, P. Aswath, A. Zhou, L. Tang, and K. T. Nguyen, J. Nanosci. Nanotechnol. 6, 1905 (2006).

${ }^{6}$ R. C. Schmidt and K. E. Healy, J. Biomed. Mater. Res. Part A 90A, 1252 (2009).

${ }^{7}$ T. B. F. Woodfield, S. Miot, I. Martin, C. A. van Blitterswijk, and J. Riesle, Biomaterials 27, 1043 (2006).

${ }^{8}$ K. Von Der Mark, V. Gauss, H. Von Der Mark, and P. Muller, Nature 267, 531 (1977).

${ }^{9}$ S. Miot, T. Woodfield, A. U. Daniels, R. Suetterlin, I. Peterschmitt, M. Heberer, C. A. van Blitterswijk, J. Riesle, and I. Martin, Biomaterials 26, 2479 (2005).

${ }^{10}$ J. Muys, M. Alkaisi, D. Melville, J. Nagase, P. Sykes, G. Parguez, and J. Evans, J. Nanobiotechnol. 4, 1 (2006).
${ }^{11}$ V. Nock, L. Murray, F. Samsuri, M. M. Alkaisi, and J. J. Evans, Microelectron. Eng. 88, 1828 (2011).

${ }^{12}$ A. Folch, B.-H. Jo, O. Hurtado, D. J. Beebe, and M. Toner, J. Biomed. Mater. Res. 52, 346 (2000).

${ }^{13}$ V. N. Goral, Y.-C. Hsieh, O. N. Petzold, R. A. Faris, and P. K. Yuen, J. Micromech. Microeng. 21, 017002 (2011).

${ }^{14}$ E. Yoshii, J. Biomed. Mater. Res. 37, 517 (1997).

${ }^{15}$ P. B. Vanwachem, A. H. Hogt, T. Beugeling, J. Feijen, A. Bantjes, J. P. Detmers, and W. G. Vanaken, Biomaterials 8, 323 (1987).

${ }^{16}$ G. Vlatakis, L. I. Andersson, R. Muller, and K. Mosbach, Nature 361, 645 (1993).

${ }^{17}$ Y. Wang, J. Balowski, C. Phillips, R. Phillips, C. E. Sims, and N. L. Allbritton, Lab Chip 11, 3089 (2011). 